\title{
Characterization of the Nurr1 ligand-binding domain co-activator interaction surface
}

\author{
Nikolaos Volakakis, Michal Malewicz, Banafsheh Kadkhodai, Thomas Perlmann \\ and Gerard Benoit \\ Ludwig Institute for Cancer Research, Stockholm Branch, Box 240, S-171 77 Stockholm, Sweden \\ ${ }^{1}$ Department of Cell and Molecular Biology, Karolinska Institute, S-171 77 Stockholm, Sweden \\ (Requests for offprints should be addressed to T Perlmann; Email: thomas.perlmann@licr.ki.se)
}

G Benoit is now at CNRS UMR 5161, Laboratoire de Biologie Moléculaire de la Cellule, IFR128 BioSciences Lyon-Gerland, École Normale Supérieure de Lyon, 46 Allée d'Italie, 69364 Lyon Cedex 07, France

\begin{abstract}
The recently solved crystal structure of the orphan nuclear receptor (NR) Nurr1 ligand-binding domain (LBD) showed that Nurr1 lacks a cavity for ligand binding and a canonical NR co-activator-binding site. Computer modeling of the Nurr1 LBD structure identified a hydrophobic region on the surface of the Nurr1 LBD that was positioned on the opposite side from the classical co-activator-binding site. Site-directed mutagenesis demonstrated that this region is critical for the activity of the Nurr1 LBD. Most mutations introduced in this region reduced or abolished transcriptional activity of the Nurr1 LBD, but mutation at lysine (K577) resulted in a drastically increased activity. Moreover, the activity of the Nurr1 LBD was shown to correlate with a propensity for proteasome-dependent degradation revealing a close association between activity and Nurr1 protein turnover. These data provide novel insights into the mechanisms of transcription via the Nurr1 LBD and identify an alternative co-activator-binding surface that is unique to the NR4A family of NRs.
\end{abstract}

Journal of Molecular Endocrinology (2006) 37, 317-326

\section{Introduction}

Nuclear receptors (NRs) comprise a large family of transcription factors that are critical during development and adult physiology. NRs include steroid hormone receptors and receptors for other lipophilic ligands such as vitamin D3, thyroid hormone, and retinoids (Mangelsdorf et al. 1995). NRs encompass several distinct functional domains. A highly conserved DNA-binding domain is utilized for specific DNA binding to hormone-response elements localized in the promoters of regulated genes and a somewhat less conserved ligand binding domain (LBD) is localized in the C-terminal region of NRs. Extensive functional and structural analyses have revealed how NR LBDs bind ligands and how the LBD can mediate both repression and activation of transcription. Accordingly, in the absence of ligands, many NRs interact with co-repressor proteins that effectively block transcription ( $\mathrm{Xu}$ et al. 1999). Ligand binding induces a conformational transition involving repositioning of the C-terminal amphipathic $\alpha$-helix (H) 12 of the canonical LBD structure (Moras \& Gronemeyer 1998). Together with other regions of the ligand-activated LBD, H12 forms a well-defined surface (activation function 2; AF-2) recognized by co-activator proteins utilizing the alleged LXXLL-motifs within amphipathic $\alpha$-helices for NR LBD recognition (Nolte et al. 1998, Shiau et al. 1998). These proteins are key components of multiprotein complexes and mediate NR-dependent gene activation by inducing histone acetylation and other chromatin modifications (Xu et al. 1999). In addition to AF-2, several NRs contain a less conserved and a poorly characterized constitutive activation domain (AF-1) within their N-terminal domains.

Several members of the NR superfamily lack identified ligands and are therefore referred to as orphan receptors (Giguere 1999). Nurr1 (NR4A2) is an orphan receptor, which together with nerve growth factor-induced clone B (NGFI-B) (NR4A1) and Nor1 (NR4A3), constitutes the NR4-family of NRs (Committee 1999). Nurr1 is expressed within the developing central nervous system where it plays key roles, for example, for the development of dopamine neurons (Perlmann \& Wallen-Mackenzie 2004). Nurr1 continues to be expressed in the adult brain and may be critical for dopamine neuron survival (Le et al. 1999), a function of significance in Parkinson's disease, which is characterized by progressive loss of dopamine neurons in the substantia nigra pars compacta. Nurr1, NGFI-B, and Nor1 are unique within the NR superfamily encoded by immediate early genes that are rapidly induced when cells are exposed to growth factors and many other stimuli.

DOI: $10.1677 / j m e .1 .02106$ Online version via http://www.endocrinology-journals.org 
Nurr1 and its closely related NR4-relatives bind to DNA either as monomers or homodimers and promote constitutive activation of transcription (Wilson et al. 1993, Maira et al. 1999). The retinoid X receptor (RXR) is a common heterodimer partner of many liganddependent NRs, including retinoic acid and peroxisome proliferators-activated receptors. In addition, Nurr1 and NGFI-B, but not Nor1, can form heterodimers with the RXR (Forman et al. 1995, Perlmann \& Jansson 1995); however, a distinguishing property of Nurr1/NGFI-B-RXR heterodimers is their ability to promote very strong transcriptional activation after binding RXR ligands like 9-cis retinoic acid (Perlmann \& Jansson 1995) or fatty acids such as docosahexaenoic acid (de Urquiza et al. 2000). In addition, Nurr1 can promote constitutive activation as a monomer or homodimer. Such activation has been shown to be strongly dependent on cell type (Castro et al. 1999).

The recent elucidation of the Nurrl LBD structure using X-ray crystal studies (Wang et al. 2003) has shown that Nurrl lacks hydrophobic pocket within its putative LBD. Instead, hydrophobic amino acid side chains occupy the region in the Nurr1 LBD that corresponds to the ligand-binding pocket in other NRs and thus prevent the capacity for ligand binding. Solving the crystal structure of the Nurr1 LBD also revealed that it is folded in a conformation resembling ligand-activated NR LBDs. Thus, in Nurr1, the 'active' conformation is independent of ligand binding. Together, these data explain previous difficulties in attempts to identify a Nurr1 ligand and also provide a structural basis for Nurrl's constitutive activity.

Despite the recent progress, several observations raise important questions concerning the mechanisms whereby Nurr1 activates transcription. Most notably, several observations reveal that the Nurr1 AF-2 promotes transcriptional activation by a mechanism different from other well-characterized NRs. First, LXXLL-containing NR co-activators that have been shown to interact with other NRs do not interact with Nurr1, and are unable to enhance Nurr1's AF-2 activity (Castro et al. 1999). Second, residues corresponding to amino acids that have been shown to be important for interaction with LXXLL-containing co-activators in other NRs are poorly conserved in Nurr1. Third, mutations at critical positions in the canonical AF-2 surface do not block Nurr1's constitutive activity when introduced at the corresponding positions in the Nurr1 LBD (Castro et al. 1999). Thus, LXXLL-containing NR co-activators that have been shown to interact with other NRs do not interact with Nurr1, and are unable to enhance Nurr1's AF-2 activity.

Collectively, these observations indicate that Nurr1 (and possibly NGFI-B and Nor1) interacts with unique co-activators and utilizes an alternative LBD region for transcriptional activation. In this study, we have used structural modeling to identify an alternative co-activator interaction surface in the Nurr1 LBD. Combined with site-directed mutagenesis, these studies defined a region that is critical for transcriptional activation within the Nurr1 LBD. This surface is likely important for interaction with Nurrl, yet unknown co-activator.

\section{Materials and methods}

\section{Plasmids}

The luciferase reporters used in transfection experiments contain three copies of the NGFI-B-binding response element (NBRE) or four copies of the Gal4binding site respectively, cloned upstream of the herpes simplex virus thymidine kinase gene minimal promoter (Perlmann \& Jansson 1995). pCMX-Nurrl contains the Nurr1 coding cDNA cloned into pCMX. pCMX-Nurr1 (85-598) was described by Nordzell et al. (2004). pCMX-Gal4 Nurr1 and pCMX-Gal4 NGFI-B contain the sequences encoding the LBDs of Nurr1 and NGFI-B respectively, cloned in frame after the sequence encoding the DNA-binding domain of yeast Gal4 (amino acids 1-147). Mutations of the different residues within Nurr1 ligand-binding domain were introduced using the Gene Editor in vitro site-directed mutagenesis kit (Promega) to generate the mutated full-length or Gal4 Nurr1 derivatives. Mutagenesis was verified by direct sequencing. The expression vector encoding RXR contains the full-length coding sequence of the human RXR $\alpha$. Detailed cloning procedure is available on request.

\section{Cell culture and transfections}

Human embryonic kidney (HEK) 293 cells and human chorion carcinoma JEG-3 cells were maintained in Dulbecco's modified Eagle's medium and minimum essential medium (Invitrogen) respectively. Media were supplemented with $10 \%$ heat-inactivated fetal calf serum (FCS) at $37^{\circ} \mathrm{C}$ under $5 \% \mathrm{CO}_{2}$ humidified atmosphere. Transfections were done using Lipofectamine Plus reagent (Invitrogen) according to the manufacturer's recommendations. Typically, $0 \cdot 4 \mu \mathrm{g}$ DNA (100 ng reporter plasmid, $100 \mathrm{ng}$ expression vector, and $200 \mathrm{ng} \beta$-galactosidase control plasmid) per well was transfected into cells in 24-well plates for 3$5 \mathrm{~h}$, after which the cells received fresh medium containing FCS (10\% final concentration) and chemicals as indicated. Cells were cultured for an additional $20 \mathrm{~h}$ and lysed by the addition of lysis buffer $(0 \cdot 65 \%$ NP40, $10 \mathrm{mM}$ Tris-HCl pH 8.0, 1 mM EDTA pH $8 \cdot 0,0 \cdot 4 \mathrm{mM}$ phenylmethylsulfonyl fluoride, and $1 \mathrm{mM}$ dithiothreitol). The cell extracts were assayed for luciferase and $\beta$-galactosidase activity using Lucy 
luminometer (Anthos Labtec Instruments, Salzburg, Austria). The ratio of luciferase activity to $\beta$-galactosidase activity in each sample was calculated to normalize the luciferase value. Each transfection was done in triplicate or quadruplicate, and the experiment was repeated at least three times. Results show the mean \pm s.E.M. of representative experiments.

\section{Western blot analysis}

Steady-state expression level of the indicated proteins was analyzed by western blot. The amount of protein loaded was normalized by $\beta$-galactosidase value to avoid transfection efficiency linked differences. Protein extracts were loaded on $10 \%$ SDS-PAGE and blotted onto polyvinylidene fluoride membranes (Bio-Rad). After transfer, membranes were washed once with PBS and saturated with $5 \%$ non-fat dry milk in PBS, $\mathrm{pH} 7 \cdot 6$, $0 \cdot 1 \%$ Tween 20 (PBS-T) for $1 \mathrm{~h}$ at room temperature. Membranes were then incubated with a specific antiserum raised against the indicated protein (Santa Cruz Biotechnology, Santa Cruz, CA, USA) in PBS-T $0.5 \%$ milk for $18 \mathrm{~h}$ at $4{ }^{\circ} \mathrm{C}$. Membranes were incubated with horseradish peroxidase-coupled antibody (Pierce, Rockford, IL, USA) for $60 \mathrm{~min}$ at room temperature. Each of these steps was followed by three washes for 10 min in PBS-T 0.5\% milk. Labeling was performed as described in the enzymatic chemiluminescence (ECL) Plus detection kit (Amersham).

\section{Results}

\section{Identification of a putative Nurr1 co-regulator-binding site}

As explained above, the recent X-ray crystal structure of the Nurr1 LBD suggested that this NR lacks a classical co-regulator-binding site (Wang et al. 2003). In other receptors, a co-activator-binding surface responsible for the interaction with LXXLL-containing co-regulators is comprised of hydrophobic amino acid side chains in H3, H4, and H12 (Fig. 1A). In contrast, the corresponding region of Nurr1 consists of polar and bulky amino acid side chains suggesting that this region is not interacting with LXXLL-containing co-activators, an observation supported by previous mutagenesis of the Nurr1 LBD (Castro et al. 1999). We used electrostatic and hydrophobic potential mapping of the Nurr1 LBD to search for other surfaces that might form a non-polar hydrophobic groove suitable for protein-protein interaction (Fig. 1B). At the opposite side of the Nurr1 LBD, amino acids L570, F574, K577 in H11, F592, L593 in H12, and L596, P597, F598 in the C-terminal protruding end of the protein form a cleft that is largely hydrophobic (Fig. 1D). We used site-directed mutagenesis to assess the functional importance of these residues. Alanine substitutions or deletions were introduced either into the Gal4 Nurr1 or the full-length Nurr1. Gal4 Nurr1 is a fusion protein containing the DNA-binding domain of the yeast transcription factor Gal4 fused to the LBD of Nurr1. The ability of these mutated derivatives to activate Gal4 or Nurr1-responsive reporter genes was examined after transfection of HEK 293 cells. Notably, when the identified hydrophobic amino acid residues were mutated, reporter gene activation was essentially abolished with the exception of the L570A mutant that exhibited only reduced activity (Fig. 2A). All mutants were expressed at similar levels in transfected HEK 293 cells as demonstrated by western blot analysis (Fig. 2B). When the corresponding residues were mutated within the full-length Nurr1, a decreased activity was also observed (Fig. 2C); however, the reduction was less dramatic due to the intact activity of AF-1 localized at the N-terminal domain of Nurr1. Taken together, these results suggest that the identified hydrophobic surface represents a co-regulator-binding site that is important for the recruitment of a yet unknown Nurrl coregulator. This conclusion is supported by a recent study that identified the similar Nurr1 LBD surface as a region interacting with peptides containing amphipathic a-helices (Codina et al. 2004).

\section{Nurr1/RXR heterodimerization affected by H11-H12 mutations}

In addition to its monomeric activity on NBREs, Nurr1 forms heterodimers with RXR that are efficiently activated by RXR-specific ligands (Perlmann \& Jansson 1995). Nurr1-RXR and heterodimers formed between RXR and other NRs have been shown to utilize a common dimer interface (Perlmann \& Jansson 1995, Aarnisalo et al. 2002). In contrast to the canonical AF-2 co-activator-binding cleft localized on the opposite side of the LBD, the newly identified Nurr1 co-regulatorbinding site is in close proximity to the RXR heterodimerization interface (Fig. 1B) suggesting that mutations affecting Nurrl monomer activity may also affect the ability of these derivatives to form heterodimers with RXR. Indeed, all Gal4-Nurr1 derivatives with activity-reducing mutations showed a significantly reduced transcriptional activity after RXR-ligand (SR11237) treatment (Fig. 3) of transfected HEK 293 cells. Co-expression of RXR in transfected cells partially rescued the reduced ligand-dependent activation of mutated Gal4 Nurr1 LBD derivatives arguing that the mutations reduced, but not totally abolished RXR heterodimerization (Fig. 3, right panel). Collectively, these data suggest that the Nurr1 co-activator 
A

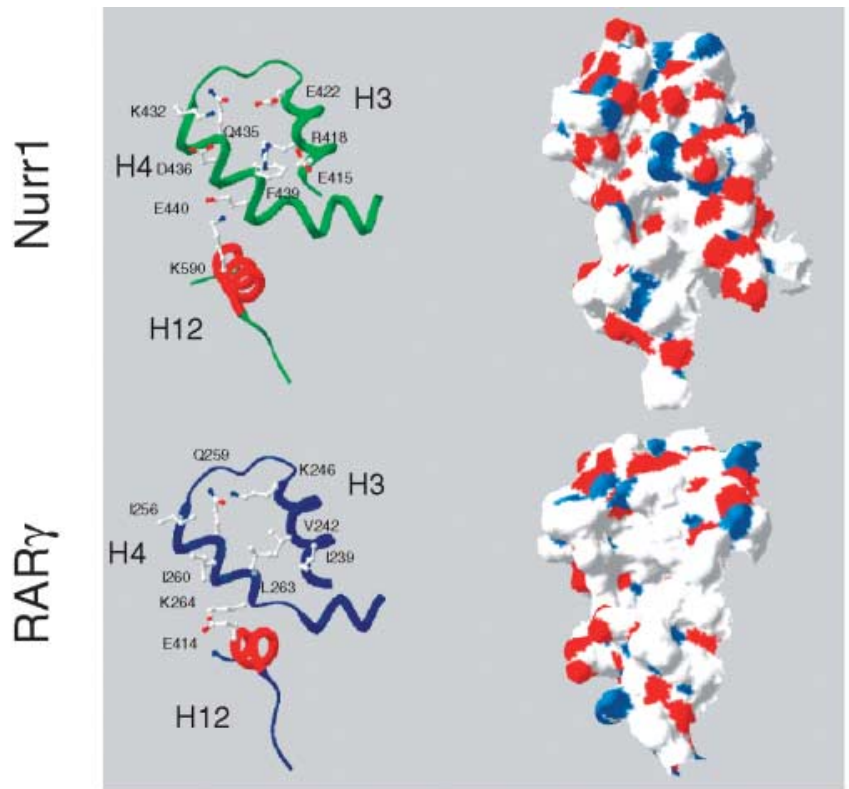

B

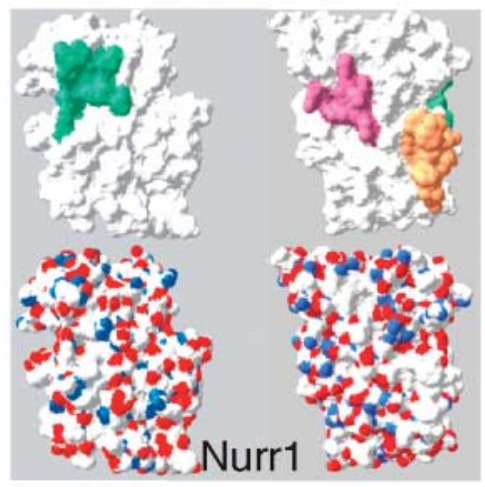

C
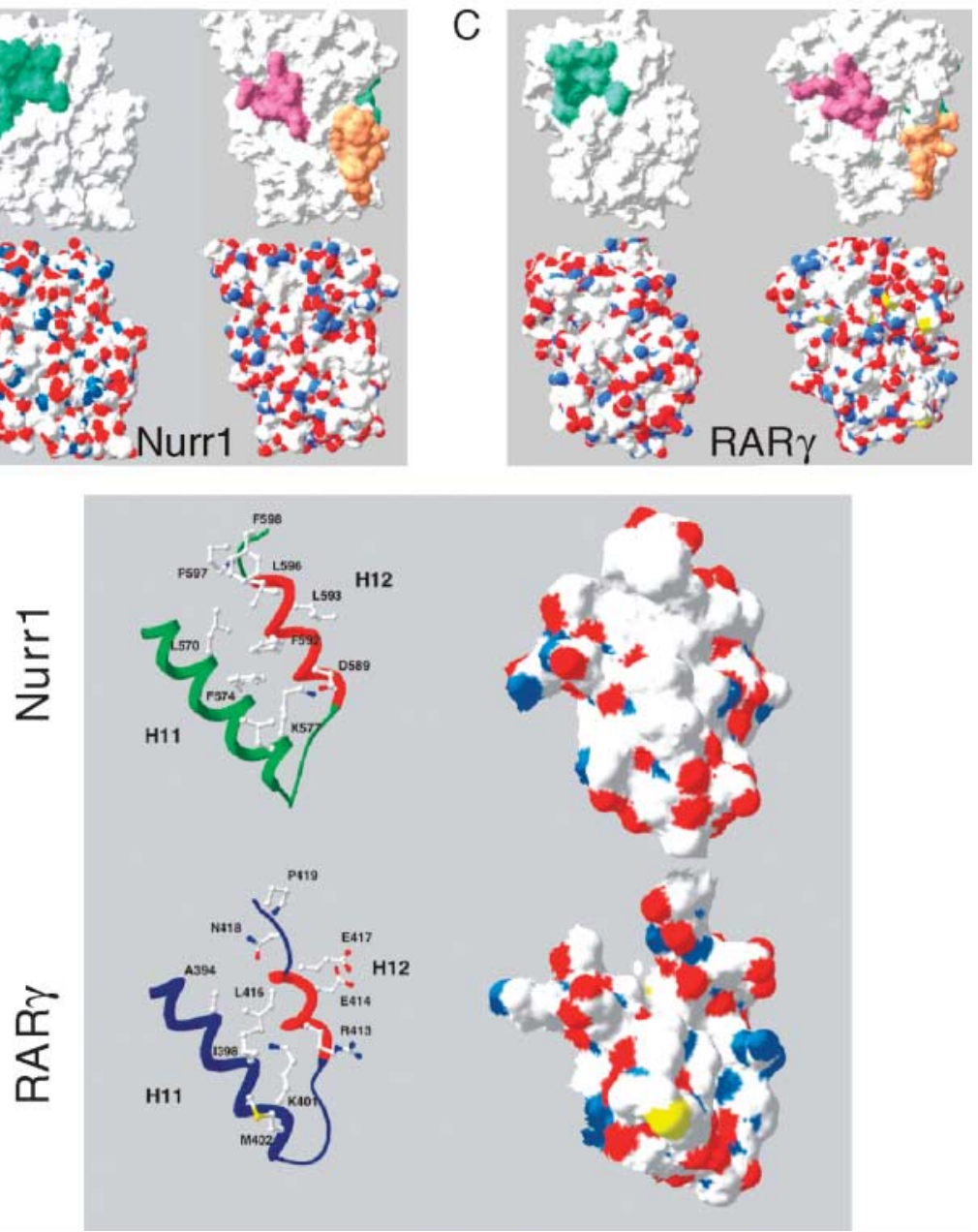
interaction surface is largely overlapping with the RXRdimer interface within the Nurr1 LBD.

\section{Hyperactive K577A mutation of Nurr1 LBD activates transcription in cell-type-independent manner}

In contrast to other Gal4-mutant derivatives, substitution of K577 to an alanine residue (Gal4 Nurr1 K577A) dramatically increased the Nurr1 LBD transcriptional activity (Fig. 4A). A corresponding increase was also observed when the mutation was introduced in the full-length Nurr1 (data not shown). In contrast, a substitution of K577 into an arginine (K577R) resulted in significantly (50\%) reduced activity (Fig. 4A). Previous studies have shown that Nurr1 LBD activity is strictly cell-type dependent. Thus, while being highly active in HEK 293 cells, Nurr1 LBD is almost inactive in human chorion carcinoma JEG-3 cells (Castro et al. 1999). Strikingly, we noted that the Gal4 Nurr1 K577A derivative was strongly active also in JEG-3 cells (58fold) demonstrating that the hyperactivity does not show the similar cell-type dependency as compared with the intact Nurr1 LBD (Fig. 4B).

\section{Nurr1 transcriptional activity is linked to decreased protein stability}

We speculated that the increased activity of the K577A derivative could be due to increased protein stability. However, unexpectedly, the steady-state expression level of the hyperactive Gal4 Nurr1 K577A derivative was dramatically decreased as compared with Gal4 Nurrl as determined by western blot of extracts from transfected HEK 293 cells (Fig. 5A). Thus, increased activity cannot be explained by higher levels of Nurr1 expression in transfected cells. The decreased steadystate level of Gal4 Nurr1 K577A could be due to either decreased synthesis or, alternatively, increased protein turnover. To test if increased turnover is responsible for the decreased levels of mutant Nurr1 derivative, transfected cells were treated with the proteasome inhibitor MG132. While the expression level of Gal4 Nurr1 was not significantly affected by MG132 treatment, the steady-state expression level of Gal4 Nurr1 K577A strongly increased (Fig. 5B). Thus, the drastically decreased expression level of the K577A mutant seems to be associated with increased proteasomedependent turnover.

The results suggest that increased turnover of Nurr1 protein is directly linked to its intrinsic transcriptional activity. Thus, introduction of a second mutation in the K577A derivative that negatively influences its transcriptional activity should stabilize the protein due to a decreased proteasome-dependent protein turnover. To test this possibility, we made an additional derivative of Gal4 Nurr1 K577A in which the 'hyperactivity' mutation was combined with a deletion of the F598 residue in a double mutant (Gal4 Nurr1 K577A/delF598). This derivative was inactive in reporter gene assays, despite the presence of the 'hyperactive' K577A substitution (Fig. 5D). Strikingly, the steady-state expression level of the double mutant was strongly increased as compared with the K577A single mutant when analyzed in extracts from transfected cells (Fig. 5A). These data provide evidence indicating that increased Nurrl turnover is tightly linked to its ability to activate transcription. Similar observations were also made in the context of the full-length Nurr1 or Nurr1 lacking the N-terminal transactivation domain (Fig. 5A (lower panel) and C).

\section{Isotype-specific activities by members of the NR4A family of NRs}

Nurr1 is a member of the NR4A family of NRs. We wished to understand if the closely related NRs of this subfamily shared the properties of Nurr1 LBD in transcriptional activation. Sequence alignment of the C-terminal region of these NRs indicated that NGF1-B differs from both Nurr1 and Nor1 in 5 out of the 30 C-terminal amino acids of $\mathrm{H} 11$ and $\mathrm{H} 12$ (Fig. 6A). Consistent with the divergence in amino acid composition, the LBD of NGFI-B is much less active compared with Nurr1 and Nor1 (Castro et al. 1999), suggesting that amino acid differences within the critical region of H11 and H12 may be responsible for their different activities. To test this possibility, a chimeric protein (NGFI-B/Nurr1-Cterm) was made containing most of the NGFI-B LBD, but with the C-terminal region replaced with that of Nurr1 (Fig. 6A). Indeed, the NGFI-B/Nurr1-Cterm derivative activated a reporter gene more efficiently as compared with wild-type NGFI-B in transfected HEK 293 cells (Fig. 6B).

Figure 1 Identification of a novel putative co-activator-binding surface in Nurr1 LBD. (A) Structural comparison of RAR $\gamma$ LBD co-activatorbinding surface and corresponding region in Nurr1 LBD. Ribbon structures shown on the left represent helices $\mathrm{H} 3, \mathrm{H} 4$, and $\mathrm{H} 12$ of the LBDs. Structures on the right show hydrophobic and electrostatic potential of the surface (white residues represent hydrophobic amino acids, red residues represent positively charged amino acids, and blue residues represent negatively charged amino acids). (B) The Nurr1 LBD and (C) the RAR $\gamma$ LBD surface respectively. Top panels show the LBD surface with the classical co-activator-binding surface (green), the novel hydrophobic surface (orange), and the RXR-binding surface (purple). Bottom panels show the hydrophobic and electrostatic potential of the LBD surface. Color coding as in (A), yellow residues represent methionine polar S atoms. Right panels depict the LBD rotated vertically by $90^{\circ}$. (D) Ribbon structures on the left show the novel putative co-activator-binding surface in Nurr1 LBD composed of helices $\mathrm{H} 11$ and $\mathrm{H} 12$ and the $\mathrm{C}$-terminal protruding end and the corresponding surface in RAR $\gamma$. Structures on the right show hydrophobic and electrostatic potential of the putative co-activator-binding site surface. Color coding of amino acids, same as in (B) and (C). 


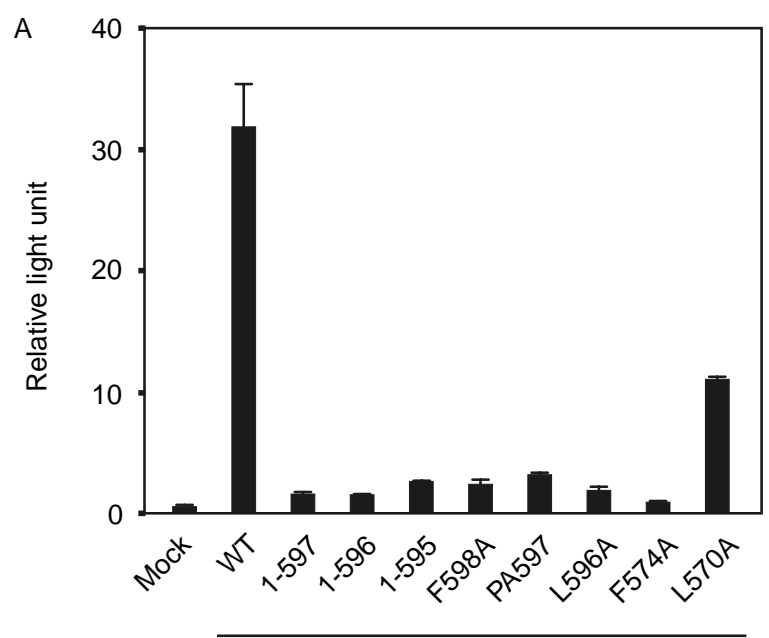

Gal4 Nurr1

B

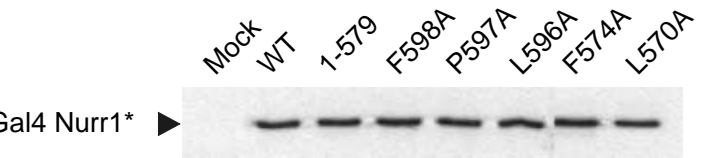

C

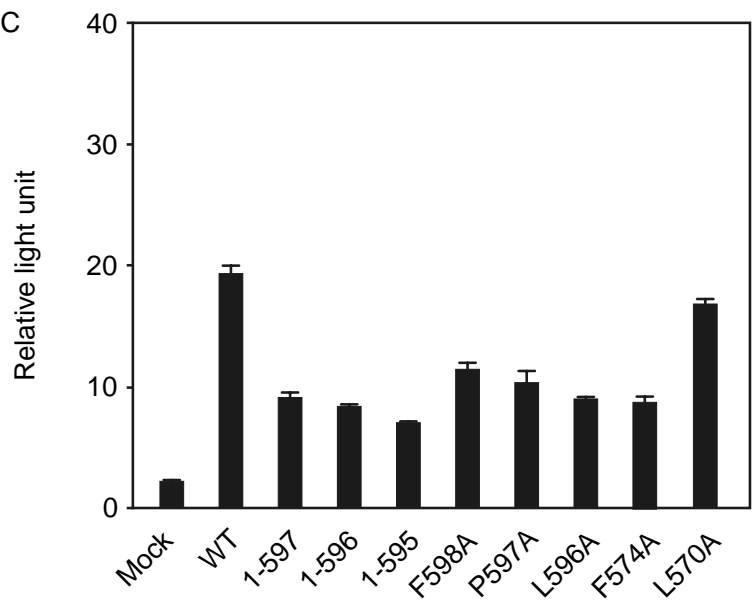

Nurr1

Figure 2 Mutations in the putative Nurr1 co-activator-binding surface impair transcriptional activity of Nurr1 AF-2. (A) Transcriptional activity of wild-type (WT) Gal4 Nurr1 LBD fusion protein and mutated derivatives in HEK 293 cells. Cells were transfected with Gal4 expression plasmids and a UAS luciferase reporter plasmid (upstream alternative sequence (UAS)-tk-luc). Luciferase activity shown on the graph was normalized to co-transfected $\beta$ galactosidase control plasmid. (B) Western blot showing steadystate protein levels of Gal4 Nurr1 and mutated derivatives. Nuclear extracts were resolved on SDS-PAGE and probed with anti-GAL4 antibody. (C) Transcriptional activity of WT Nurr1 and mutated derivatives in HEK 293 cells. Cells were transfected with Nurr1 expression plasmids and a NBRE luciferase reporter plasmid (NBRE-tk-luc). Luciferase activity presented as in (A).

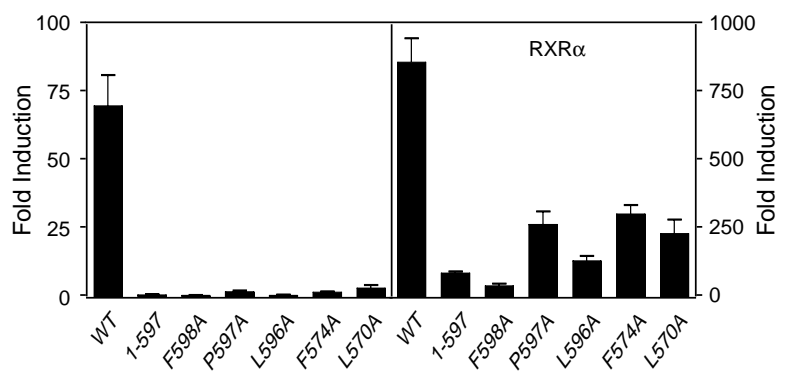

Figure 3 Mutations in the putative Nurr1 co-activator-binding surface affect the ability of Nurr1 LBD to respond to RXR ligands. Transcriptional activity of Gal4 Nurr1 and mutated derivatives after treatment with RXR-specific ligands, without (left panel) or with (right panel) co-transfected RXR. The bars represent fold induction calculated by dividing the reporter gene activity in the presence of RXR agonist by the activity measured in the vehicletreated cells. HEK 293 cells were transfected with Gal4 expression plasmids and UAS-tk-luc without (left panel) or with (right panel) $\mathrm{RXR} \alpha$ expression vector and treated with vehicle control or RXR ligand overnight. Luciferase activity presented as in Fig. 2.

However, wild-type Nurr1 was more active than NGFIB/Nurr1-Cterm (data not shown), indicating that additional regions outside the $\mathrm{H} 11 / \mathrm{H} 12$ region also play critical roles in transcriptional activation. Nonetheless, the results demonstrate that the differences between Nurr1 and NGFI-B in H11 and H12 significantly contribute to the functional differences observed between these two proteins.

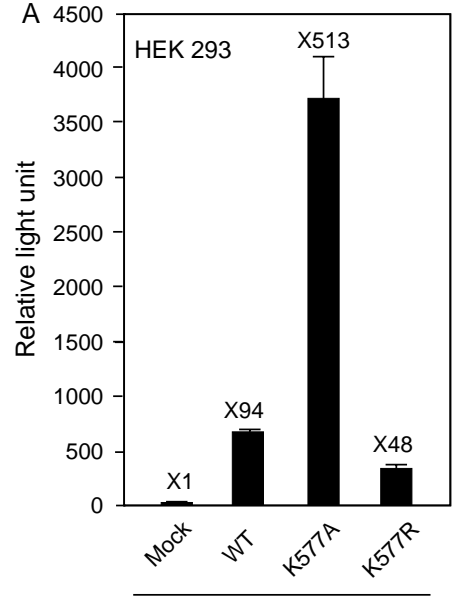

Gal4 Nurr1 LBD

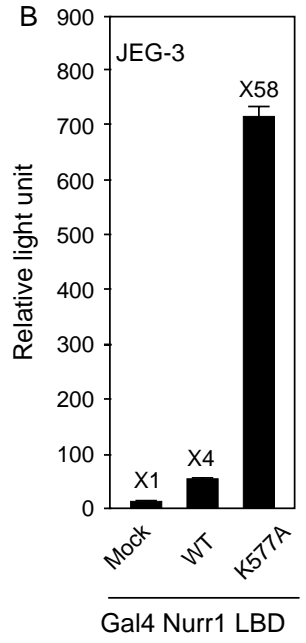

Figure 4 Mutation of lysine 577 to alanine in the Nurr1 LBD leads to increased transcriptional activity. (A) Transcriptional activity of WT Gal4 Nurr1 LBD fusion protein and mutated derivatives in HEK 293 cells. Cells were transfected with Gal4 expression plasmids and UAS-tk-luc. Luciferase activity presented as in Fig. 2. Fold activation is depicted above the bars. (B) Experiment performed as in (A) in JEG-3 cells. 


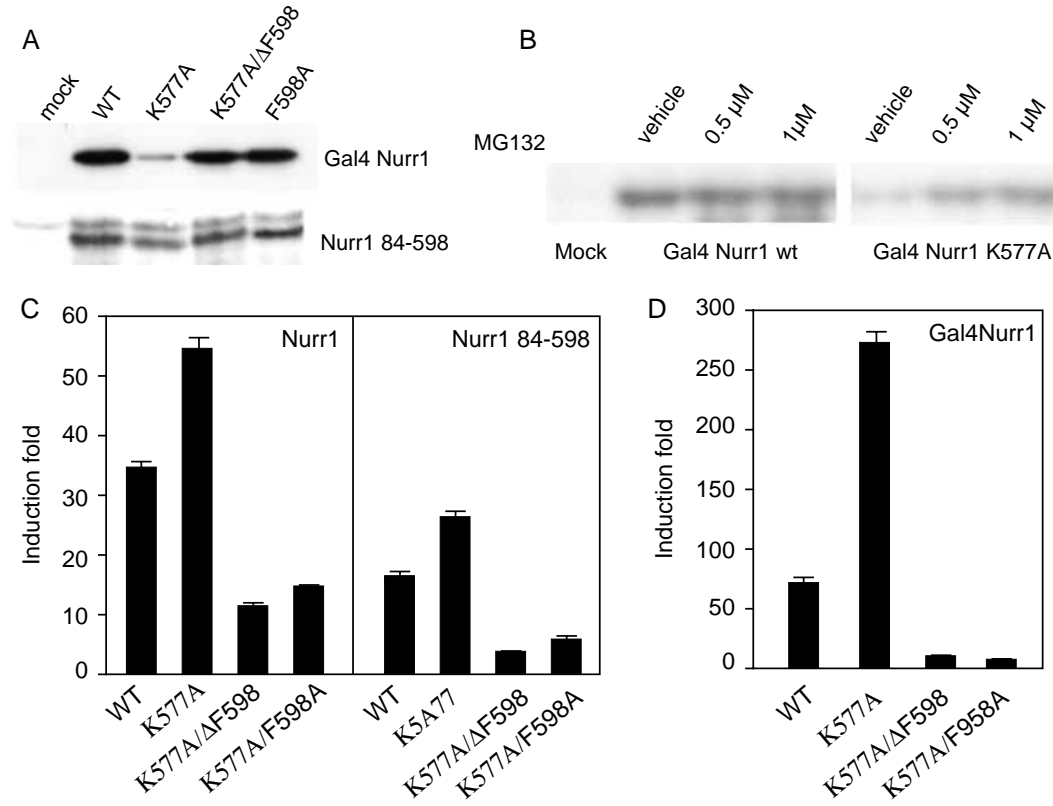

Figure 5 Mutation of lysine 577 to alanine in the Nurr1 LBD leads to decreased steadystate protein levels due to transcription-linked enhanced proteosomal-mediated turnover. Western blot showing steady-state protein levels of Gal4 Nurr1 and mutated derivatives (upper panel) or steady-state protein levels of AF-1-deleted Nurr1 and mutated derivatives (lower panel). HEK 293 cells were transfected with Gal4 Nurr1 LBD expression plasmids or Nurr1 expression plasmids. Nuclear extracts were prepared after $24 \mathrm{~h}$, resolved on SDS-PAGE, and probed with anti-GAL4 antibody (upper panel) or antiNurr1 antibody (lower panel). (B) Western blot showing steady-state protein levels of Gal4 Nurr1 (left panel) and K577A derivative (right panel) in the presence or absence of indicated amounts of proteasome inhibitor MG132. HEK 293 cells were transfected with Gal4 Nurr1 LBD expression plasmids and treated with MG132 for $8 \mathrm{~h}$. Western blot was performed as in (A). (C) Transcriptional activity of Nurr1 and mutated derivatives measured in HEK 293 cells. Left panel shows the full-length Nurr1 derivatives and right panel the AF-1-deleted Nurr1 derivatives. Cells were transfected with Nurr1 expression plasmids and NBRE-tk-luc. Luciferase activity is presented as fold induction.

(D) Experiment performed as in (C) with the Gal4 Nurr1 LBD fusion protein.

\section{Discussion}

The crystal structures of the LBDs of Nurr1 and its Drosophila homolog DHR38 have highlighted unique features of these receptors. Of particular importance, these proteins lack a hydrophobic ligand-binding pocket typical for other ligand-activated NRs. Moreover, Nurr1/DHR38 apparently lacks canonical co-regulatorbinding cleft, which in other NRs is utilized for interactions with LXXLL-containing co-activators. These properties explain the previous observations showing that the members of the NR4A family are incapable of interacting with known co-activators via their LBDs (Castro et al. 1999). A previous study using a novel nuclear magnetic resonance (NMR) foot-printing approach identified a unique and highly hydrophobic surface in Nurr1 (also present in other NR4A members) that could interact with an amphipathic co-repressor/activator-derived peptide (Codina et al. 2004). In the present work, we used computer modeling combined with in vitro mutagenesis and functional assays to analyze the role of the identified hydrophobic surface.

In the analysis of the Nurr1 LBD, we introduced amino acid substitutions that, based on computer modeling, would likely affect surface properties. However, it is important to take into consideration that amino acid substitutions can also disrupt the conformation of the protein and such conformational changes might also compromise the function of Nurr1. This possibility is of particular concern for two of the identified residues (F574 in helix 11 and D589 in helix 12 ), in which both were proposed to play a role in stabilizing helix 12 in the Nurr1 LBD (Wang et al. 2003). However, for the following two reasons we do not believe that the reduced activity of these mutants is due to destabilization of the overall Nurr1 LBD structure. First, NMR analysis of the F574A mutant indicated that this mutation does not disrupt the overall conformation of the Nurr1 LBD (Codina et al. 2004). Second, D589 


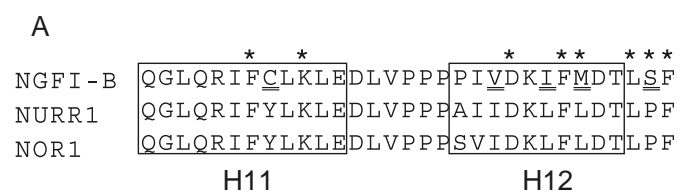

B

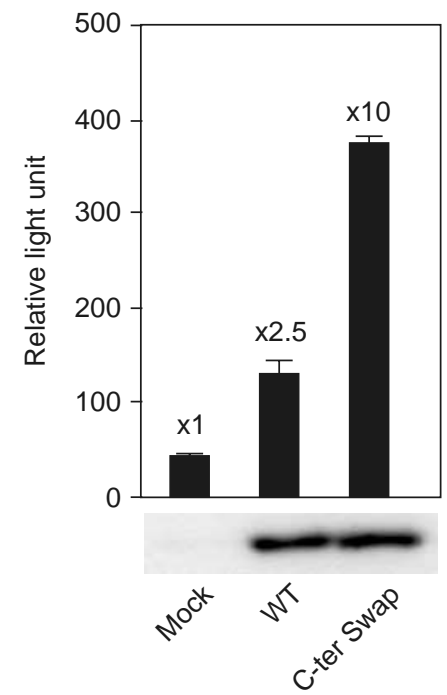

Figure 6 The AF-2 transcription-activation domain of NGFI-B nuclear receptor is inactive due to an evolutionary divergence from Nurr1 and Nor1 in the C-terminal portion of the LBD.

(A) Amino acid sequence alignment of the C-terminal end of NR4A receptors. The eight amino acids forming the novel putative Nurr1 co-activator-binding surface are indicated with a star. NGFI-B differs from Nurr1 and Nor1 at five amino acid positions (underlined). (B) Transcriptional activity of Gal4 NGFI-B LBD (WT) and a fusion protein in which the amino acid regions of NGFI-B and Nurr1 shown in (A) were swapped (C-ter swap). HEK 293 cells were transfected with Gal4 expression plasmids and UAS-tk-luc. Luciferase activity presented as in Fig. 2. Fold activation is depicted above the bars. The western blot shows the steady-state protein levels of Gal4 Nurr1 and C-terminal swapped derivative. HEK 293 cells were transfected with Gal4 Nurr1 LBD expression plasmids. Nuclear extracts were prepared after $24 \mathrm{~h}$, resolved on SDS-PAGE and probed with anti-GAL4 antibody.

forms a salt bridge with K577 (Wang et al. 2003); however, when substituting the latter residue for an alanine (which would also disrupt the salt bridge) the resulting mutated Nurrl derivative was hyperactive, thus arguing against a major destabilization of helix 12 when the salt bridge is disrupted. Instead, the most probable explanation of our findings is that both F592 and D589 are critical for the interaction with an unidentified Nurr1 co-activator.

Interestingly, the newly identified Nurr1 LBD surface overlaps with a region, which in Nurr1 and several other NRs is important for heterodimerization with RXR. It was therefore expected that most of the mutations affecting Nurr1 LBD transcriptional activity also decreased Nurr1's ability to function as a heterodimer with RXR. In contrast to our observations, a recent publication comparing the crystal structures of NGFI-B and Nurr1 LBDs found that mutations of two of the residues localized within the combined activation/heterodimer surface of the Nurr1 LBD (L596 and F598) did not affect interaction with RXR (Flaig et al. 2005). We believe that the discrepancy can be explained by minor differences in the way the functional transfection assays were performed. In our experiments, activation is detected as a consequence of endogenous RXR binding to Gal4 Nurr1 resulting in a robust transactivation upon addition of RXR ligand. Thus, in contrast to the experiments reported by Flaig et al. (2005), reduced, albeit not abolished, RXR interactions are probably more easily detected in our experiments. Indeed, we found that most of the mutations appeared to reduce, but did not abolish, interactions with RXR (Fig. 3). This conclusion is further supported by the ability of overexpressed $\operatorname{RXR} \alpha$ to partially rescue the negative effect of Nurr1 LBD mutations (Fig. 3, right panel). We hypothesize that in this case increased RXR expression compensates the lower affinity of mutated Nurr1 LBDs to restore the interaction with RXR.

Previous studies identified point mutations in the Nurr1 LBD that abolished Nurr1-RXR heterodimerization without altering the ability of Nurrl to activate transcription as a monomer (Aarnisalo et al. 2002). In accordance with the close proximity of the hydrophobic transactivation patch and heterodimerization surface, the data in this study demonstrated that several mutations affecting Nurr1 monomeric activity also destabilized RXR-Nurr1 heterodimerization. Moreover, some of the mutations affecting Nurrl-RXR heterodimerization increased the ability of Nurr1 to activate as a monomer (Aarnisalo et al. 2002). Together, these observations suggest that RXR and a Nurrl-specific co-activator interact with distinct but overlapping binding surfaces, thus suggesting that RXR and co-activator binding to the Nurr1 LBD may be mutually exclusive and explaining why RXR overexpression in transfected cells results in diminished Nurr1 monomeric activity (Aarnisalo et al. 2002). Therefore, the ability of co-activator-bound Nurr1 monomers to activate gene expression in different cell types is likely influenced by the expression level of 'competing' RXR. Interestingly, Nurr1 is very efficiently activating transcription in response to RXR ligands in JEG-3 cells, presumably as a result of high levels of RXR expression in these cells. It is therefore striking that the monomeric activity of Nurrl is unusually low in this particular cell line.

A surprising result from our mutational analysis was the finding that the K577A mutant strongly increased the transcriptional activity of Nurr1 both in HEK 293 and human chorion carcinoma JEG-3 cells in which the wild-type Nurr1 LBD is virtually inactive (Fig. 4). Abolished interactions with co-repressors might explain the increased transcriptional activity of the K577A 
mutant. However, in various pull-down experiments, we were unable to show any direct physical interaction of silencing mediator of retinoid and thyroid receptors (SMRT) co-repressors with Nurr1 LBD (data not shown) arguing against the involvement of classical NR co-repressors in the regulation of Nurrl activity. Instead, we hypothesize that the Nurr1 interacts with co-activators and the mutation disrupts such interactions. The type of the residue 577 (charged versus hydrophobic) could determine the strength of interaction with unknown co-activators. It is also possible that $\mathrm{K} 577$ is post-translationally modified in a way that influences Nurrl transcriptional activity. In a recent report (Galleguillos et al. 2004), Nurr1 was shown to interact with the SUMO (small ubiquitin-like modifier)-E3 ligase protein inhibitor of activated STAT protein $\gamma$ (PIAS $\gamma$ ). Interestingly, the $\mathrm{K} 577$ residue in Nurr1 LBD is part of a consensus SUMO-modification sequence and mutation of this lysine to arginine (which cannot be SUMO modulated) resulted in decreased activity, suggesting that sumoylation of K577 is potentially important for Nurr1 transcriptional activity. However, our observation that an alanine substitution (which also cannot be SUMOylated) strongly increased activity argues against the simple explanation that the SUMOylated state of this residue would determine Nurrl's transcriptional activity.

The enhanced proteasome-dependent turnover of the K577A derivative suggests that the transcriptional activity of Nurrl is coupled to degradation (Fig. 5). These observations resemble the regulation of retinoic acid receptor (RAR), the estrogen receptor, and the progesterone receptor (Lonard et al. 2000, Shen et al. 2001), which after ligand-induced activation, are rapidly degraded via the ubiquitin/proteasome pathway. Analysis of the molecular mechanisms explaining these observations suggest that the targeted degradation of several nuclear receptors is mediated via the ligand-dependent recruitment of SUG-1, a protein belonging to the $19 \mathrm{~S}$ regulatory subunit of the 26S proteasome (vom Baur et al. 1996). However, overexpression of SUG-1 shows no effect on Gal4 Nurr1 transcriptional activity and steady-state expression level (data not shown) suggesting an alternative mechanism for degradation of active Nurr1 protein.

In conclusion, our data has defined a unique region important for the transcriptional activation of Nurr1. As we have demonstrated, mutations in this region can both lead to disrupted or enhanced transcriptional activity. Our findings further emphasize the unique properties of the NRs belonging to the NR4A group (Nurr1, Nor1, and NGFI-B) and provide strong evidence for the existence of unique co-activators interacting with the LBDs of these NRs. A particularly interesting important future goal should be to identify those unique co-activators and elucidate how they contribute to the function of Nurr1 and its close NR relatives.

\section{Acknowledgements}

NV was supported by a scholarship from the Alexander S Onassis Public Benefit Foundation.

\section{Funding}

This work was supported by the Swedish Strategic Science Foundation (SSF) with a grant to the Center of Excellence for Developmental Biology (CEDB). The results reported in this paper do not involve conflict of interest that would prejudice its impartiality.

\section{References}

Aarnisalo P, Kim CH, Lee JW \& Perlmann T 2002 Defining requirements for heterodimerization between the retinoid $\mathrm{X}$ receptor and the orphan nuclear receptor Nurr1. Journal of Biological Chemistry 277 35118-35123.

Castro DS, Arvidsson M, Bondesson Bolin M \& Perlmann T 1999 Activity of the Nurrl carboxyl-terminal domain depends on cell type and integrity of the activation function 2. Journal of Biological Chemistry 274 37483-37490.

Codina A, Benoit G, Gooch JT, Neuhaus D, Perlmann T \& Schwabe JW 2004 Identification of a novel co-regulator interaction surface on the ligand binding domain of Nurrl using NMR footprinting. Journal of Biological Chemistry 279 53338-53345.

Committee NN 1999 A unified nomenclature system for the nuclear receptor superfamily. Cell 97 161-163.

de Urquiza AM, Liu S, Sjoberg M, Zetterstrom RH, Griffiths W, Sjovall J \& Perlmann T 2000 Docosahexaenoic acid, a ligand for the retinoid $\mathrm{X}$ receptor in mouse brain. Science $2902140-2144$.

Flaig R, Greschik H, Peluso-Iltis C \& Moras D 2005 Structural basis for the cell-specific activities of the NGFI-B and the NURR1 ligandbinding domain. Journal of Biological Chemistry 280 19250-19258.

Forman BM, Umesono K, Chen J \& Evans RM 1995 Unique response pathways are established by allosteric interactions among nuclear hormone receptors. Cell 81 541-550.

Galleguillos D, Vecchiola A, Fuentealba JA, Ojeda V, Alvarez K, Gomez A \& Andres ME 2004 PIASgamma represses the transcriptional activation induced by the nuclear receptor Nurr1. Journal of Biological Chemistry 279 2005-2011.

Giguere V 1999 Orphan nuclear receptors: from gene to function. Endocrine Reviews 20 689-725.

Le W, Conneely OM, He Y, Jankovic J \& Appel SH 1999 Reduced Nurr 1 expression increases the vulnerability of mesencephalic dopamine neurons to MPTP-induced injury. Journal of Neurochemistry 73 2218-2221.

Lonard DM, Nawaz Z, Smith CL \& O'Malley BW 2000 The 26S proteasome is required for estrogen receptor-alpha and coactivator turnover and for efficient estrogen receptor-alpha transactivation. Molecular Cell 5 939-948.

Maira M, Martens C, Philips A \& Drouin J 1999 Heterodimerization between members of the Nur subfamily of orphan nuclear receptors as a novel mechanism for gene activation. Molecular and Cellular Biology 19 7549-7557.

Mangelsdorf DJ, Thummel C, Beato M, Herrlich P, Schutz G, Umesono K, Blumberg B, Kastner P, Mark M, Chambon P et al. 1995 The nuclear receptor superfamily: the second decade. Cell 83 835-839. 
Moras D \& Gronemeyer H 1998 The nuclear receptor ligand-binding domain: structure and function. Current Opinion in Cell Biology 10 384-391.

Nolte RT, Wisely GB, Westin S, Cobb JE, Lambert MH, Kurokawa R, Rosenfeld MG, Willson TM, Glass CK \& Milburn MV 1998 Ligand binding and co-activator assembly of the peroxisome proliferatoractivated receptor-gamma. Nature 395 137-143.

Nordzell M, Aarnisalo P, Benoit G, Castro DS \& Perlmann T 2004 Defining an N-terminal activation domain of the orphan nuclear receptor Nurr1. Biochemical and Biophysical Research Communications 313 205-211.

Perlmann T \& Jansson L 1995 A novel pathway for vitamin A signaling mediated by RXR heterodimerization with NGFI-B and NURR1. Genes and Development 9 769-782.

Perlmann T \& Wallen-Mackenzie A 2004 Nurr1, an orphan nuclear receptor with essential functions in developing dopamine cells. Cell and Tissue Research 318 45-52.

Shen T, Horwitz KB \& Lange CA 2001 Transcriptional hyperactivity of human progesterone receptors is coupled to their ligand-dependent down-regulation by mitogen-activated protein kinase-dependent phosphorylation of serine 294. Molecular and Cellular Biology 21 6122-6131.
Shiau AK, Barstad D, Loria PM, Cheng L, Kushner PJ, Agard DA \& Greene GL 1998 The structural basis of estrogen receptor/coactivator recognition and the antagonism of this interaction by tamoxifen. Cell 95 927-937.

vom Baur E, Zechel C, Heery D, Heine MJ, Garnier JM, Vivat V, Le Douarin B, Gronemeyer H, Chambon P \& Losson R 1996 Differential ligand-dependent interactions between the AF-2 activating domain of nuclear receptors and the putative transcriptional intermediary factors MSUG1 and TIF1. EMBO Journal 15 $110-124$.

Wang Z, Benoit G, Liu J, Prasad S, Aarnisalo P, Liu X, Xu H, Walker NP \& Perlmann T 2003 Structure and function of Nurr1 identifies a class of ligand-independent nuclear receptors. Nature 423 555-560.

Wilson TE, Fahrner TJ \& Milbrandt J 1993 The orphan receptors NGFI-B and steroidogenic factor 1 establish monomer binding as a third paradigm of nuclear receptor-DNA interaction. Molecular and Cellular Biology 13 5794-5804.

Xu L, Glass CK \& Rosenfeld MG 1999 Coactivator and co-repressor complexes in nuclear receptor function. Current Opinion in Genetics and Development 9 140-147.

Received 12 June 2006

Accepted 10 July 2006 